\title{
Lanthanide upconversion nanocrystals within microstructured optical fibres; a sensitive platform for biosensing and a new tool for nanocrystal characterisation
}

E. P. Schartner, D. Jin, H. Ebendorff-Heidepriem, J. A. Piper, T. M. Monro

E. P. Schartner, D. Jin, H. Ebendorff-Heidepriem, J. A. Piper, T. M. Monro, "Lanthanide upconversion nanocrystals within microstructured optical fibres; a sensitive platform for biosensing and a new tool for nanocrystal characterisation," Proc. SPIE 8351, Third Asia Pacific Optical Sensors Conference, 83512Q (31 January 2012); doi: 10.1117/12.915968 


\title{
Lanthanide upconversion nanocrystals within microstructured optical fibres; a sensitive platform for biosensing and a new tool for nanocrystal characterisation
}

\author{
E P Schartner ${ }^{a}$, D. Jin ${ }^{\text {, }}$ H. Ebendorff-Heidepriem ${ }^{a}$, J. A. Piper ${ }^{\text {b }}$ T M Monro ${ }^{a}$ \\ ${ }^{\mathrm{a}}$ Institute of Photonics \& Advanced Sensing, University of Adelaide, Adelaide, Australia \\ bAdvanced Cytometry Labs, MQ Photonics Research Centre, Macquarie University, Sydney, \\ Australia \\ Email: erik.schartner@adelaide.edu.au
}

\begin{abstract}
We investigate a powerful new sensing platform based on upconversion luminescence in $\mathrm{NaYF}_{4}$ : $\mathrm{Yb} / \mathrm{Er}$ nanocrystals loaded inside a suspended-core microstructured optical fibre. The use of a NIR source enables autofluorescence from the glass to be reduced compared to using visible sources for excitation of fluorescence. We demonstrate a substantial improvement in the detection limit that can be achieved in a suspended-core fibre sensor, with detection limits as low as $660 \mathrm{fM}$ achieved. This is a factor of $15 \times$ better than the best results previously reported using Quantum dots in a similar fibre.
\end{abstract}

Keywords: microstructured optical fibre, lanthanide, biosensing, nanocrystal, fluorescence

\section{INTRODUCTION}

Applications such as environmental monitoring, food production, medical diagnostics and national security require chemical and biomolecular sensor platforms with the following attributes: 1 . The capacity to detect and characterize trace quantities of analytes; 2. Low cost and compact size with disposable sensing tips consuming small sample quantities; 3. Reliability, reproducibility and ease and speed of operation; 4. Capacity for use in high-throughput screening of multiple samples in a single test. To date, no single platform has been able to meet all of these key requirements.

One promising approach uses fluorescence based detection methods within optical fibre, offering good sensitivity [1] arising from their ability to combine long interaction lengths with small sample volumes. Previous work has identified autofluorescence as a factor that limits the sensitivity that can be achieved in fluorescence based biological or chemical sensors [1, 2]. Our recent work on dip sensors based on suspended core optical fibres has reduced the background associated with the glass host itself [1] , but further improvements would be possible if alternative labelling molecules could be used to circumvent this autofluorescence emission.

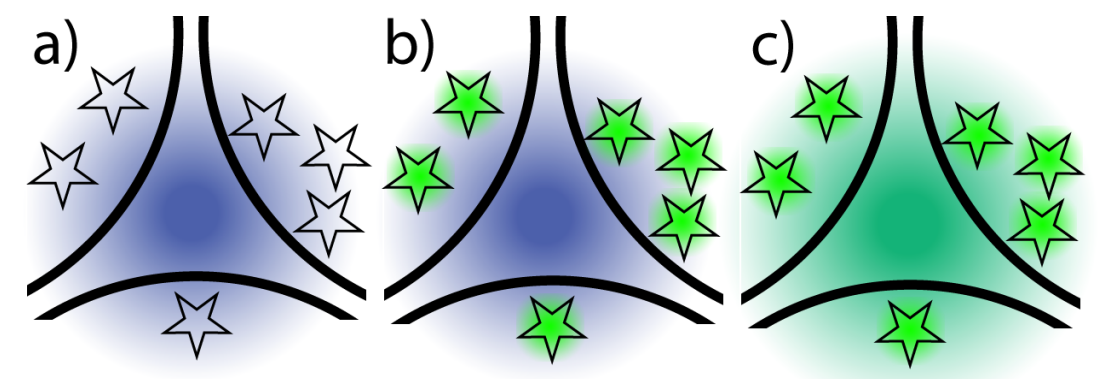

Figure 1 - Evanescent wave sensing mechanism using suspended core fibres. The region within the triangle is the fibre core, which is made of glass. By filling the holes of the fibre with the analyte, it is possible to exploit the interaction of the portion of the guided light (blue) with nanoparticles in the fluid surrounding the core of the fibre (a-b). A portion of this fluorescence (green) is then recaptured into guided modes in the fibre, and can be detected at either end (c).

Third Asia Pacific Optical Sensors Conference, edited by John Canning, Gangding Peng, Proc. of SPIE Vol. 8351, 83512Q - ( 2012 SPIE · CCC code: 0277-786X/12/\$18 · doi: 10.1117/12.915968 
Lanthanide doped upconversion nanoparticles (for example hexagonal phase $\mathrm{NaYF}_{4}: \mathrm{Yb} / \mathrm{Er}$ nanocrystals) are powerful tools for biological imaging and sensing [3-5]. These nanocrystals do not photobleach or blink, exhibit low toxicity, and offer comparatively bright, visible multi-colour emissions excited at the IR wavelength, typically at $~ 976$ $\mathrm{nm}$. These nanoparticles can be surface functionalized by either amphiphilic carboxyl-polymer or amino-silica [3] to become biocompatible [6]. They can be conjugated to specific antibodies or DNA strands as fluorescent tags to probe targeted analytes.

Of particular note, upconversion luminescence feature can provide autofluorescence-background-free conditions for ultra-sensitive biosensing applications [5, 7], since IR excitation does not stimulate intrinsic visible autofluorescence in the surrounding biosample. Moreover, since this anti-Stokes luminescence absorbs sequential secondary photons involving metastable-excited states with lifetimes in milliseconds, upconversion can be up to $10^{5}$ times more efficient than most 2-photon fluorescent dyes [3]. Furthermore, this fluorescent nanoparticle candidate exhibits exceptionally long luminescence lifetimes that range from tens to several hundreds of microseconds, through control of the crystal growth and surface conditions. This provides rich opportunities for advanced biosensing. For example, it enables the timeresolved discrimination of multiple analytes at a single test.

In this paper we explore the performance of hexagonal-phase $\mathrm{NaY}_{0.8} \mathrm{~F}_{4}: \mathrm{Yb}_{0.18} / \mathrm{Er}_{0.02}$ nanocrystals (44 nm in diameter) when they are loaded into a microstructured optical fibre with a small, suspended core [8,9]. Our experiments demonstrate that this upconversion fluorescence can be characterised using relatively low laser powers from a solid-state $980 \mathrm{~nm}$ laser diode over a large power dynamic range. This is important for consistent power-dependent characterization of this advanced functional nanomaterial. The time-resolved luminescence can be detected by a compact semiconductor photomultiplier tube (PMT), which suggests a portable low-cost biosensor platform. Moreover, we are able to show that due to the reduced background fluorescence from the glass at this pump wavelength compared to that observed previously using a visible laser; we are able to significantly reduce the detectable concentration relative to previous work based on quantum dots in similar fibres using visible $532 \mathrm{~nm}$ excitation.

\section{NANOPARTICLE CHARACTERISATION}

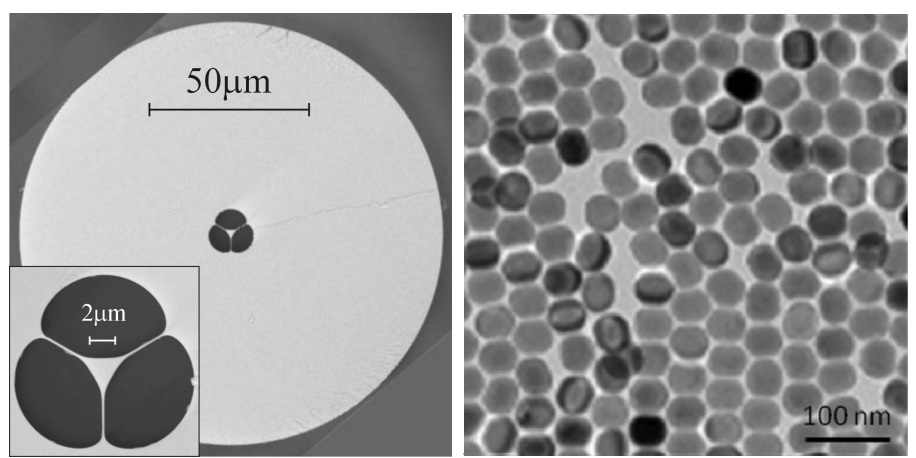

Figure 2 - Scanning Electron Microscope (SEM) image of the suspended core soft glass (F2) MOF used for these experiments. The central (core) region of the fibre was made from F2HT glass, and the cladding from F2 glass. The nanocrystal TEM (44 $\mathrm{nm}$ in diameter mono-dispersed).

Nanoparticles were prepared using a modified user-friendly synthesis method via the solvothermal route $[3,5]$ and diluted in cyclohexane or toluene. The experimental setup shown below in Figure 3 was used. Capillary action was used to fill the fibres with the solutions, with a filling time of approximately 15 minutes for $19 \mathrm{~cm}$ of fibre. The $980 \mathrm{~nm}$ laser was operated in CW mode for these measurements at incident power levels from $1 \mathrm{~mW}-300 \mathrm{~mW}$. The coupling efficiency into the fibre ranges from 10-20\% depending on the core size and coupling optics used. An example of the upconversion spectra obtained is shown in Figure 4.The characteristic emission peaks are attributed to the ${ }^{2} \mathrm{H}_{11 / 2},{ }^{4} \mathrm{~S}_{3 / 2} \rightarrow$ ${ }^{4} \mathrm{I}_{15 / 2}$ transition in the green spectral region with another red emission arising from ${ }^{4} \mathrm{~F}_{9 / 2} \rightarrow{ }^{4} \mathrm{I}_{15 / 2}$. 


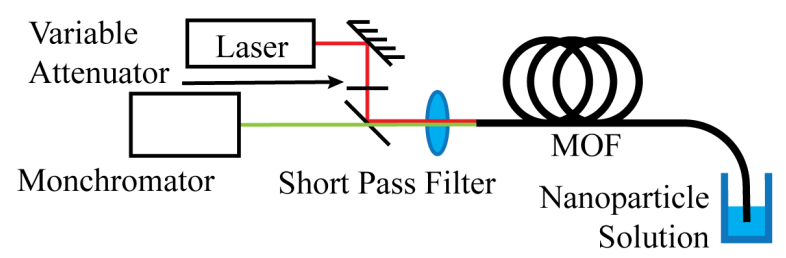

Figure 3 - Experimental configuration for characterization measurements using suspended core MOF and lanthanide nanoparticle solution with980 nm excitation. A HR @980 nm, HT @544 nm filter was used to effectively block the residual excitation light while passing the visible upconversion emission.

The fibre used for these experiments was an in-house fabricated suspended core F2 glass MOF as shown in Figure 2. The fibre was fabricated via preform extrusion [10] and subsequent fibre drawing was performed using a cane-in-tube technique to allow the production of a relatively small core within a robust fibre geometry [9]. The fabricated fibre had a core diameter of $\sim 1.7 \mu \mathrm{m}$ and an outer diameter $130 \mu \mathrm{m}$ with a hole size of $\sim 7 \mu \mathrm{m}$.

\section{LOW CONCENTRATION MEASUREMENTS}

Building on this work on characterising the emission of the nanocrystals within the suspended core fibres, measurements were performed on nanoparticles dispersed in toluene with known concentrations. Serial dilutions ranging between $66 \mathrm{nM}$ and $66 \mathrm{fM}$ were performed, and spectra obtained at different input powers. An example of one of these spectra compared to that of an unfilled fibre is shown in Figure 4 below. The experimental configuration was the same as that used in Section 2, and is shown in Figure 3. Care was taken in measurements to ensure that solvent evaporation was minimised during fibre filling.

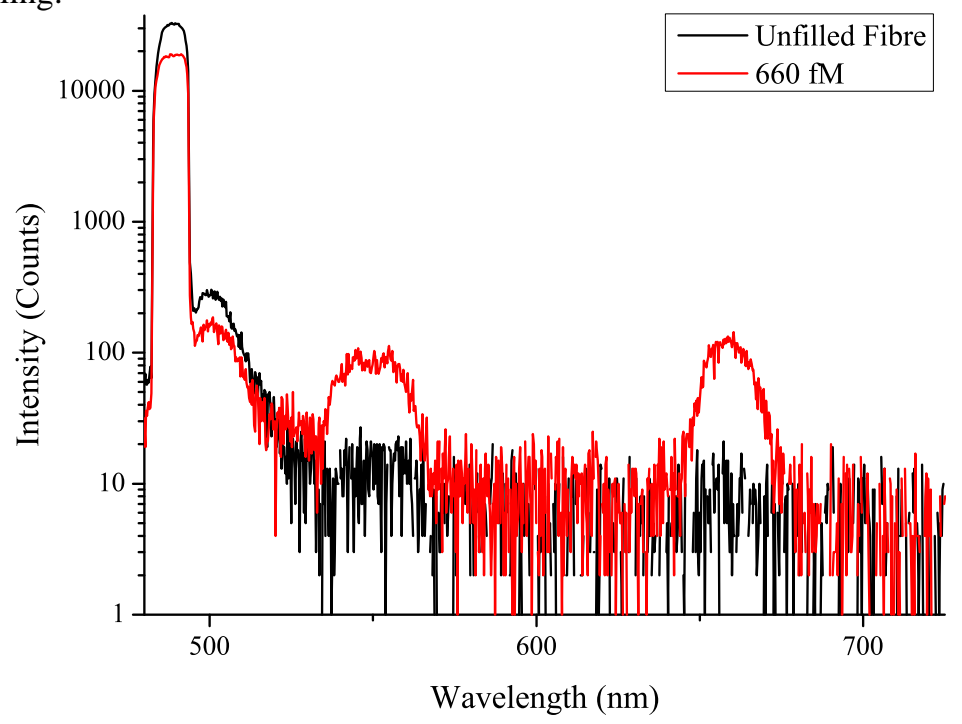

Figure 4 - Differential fluorescence spectra between unfilled fibre (black) and fibre filled with a $660 \mathrm{fM}$ concentration of nanoparticles. The excitation powers and fibre lengths are identical for both cases.

This result demonstrates that the nanoparticles are still detectable even at the relatively low concentration of $660 \mathrm{fM}$, which corresponds to approximately 11000 particles in a $19 \mathrm{~cm}$ length of fibre with a total volume of $29 \mathrm{~nL}$. This is limited again by background fluorescence from the glass, with longer lengths of fibre showing a clearer signal than shown here. The position of this fluorescence strongly suggests the presence of erbium within the glass. When measurements are taken with no solvent, or alternatively when the fibre is filled with toluene (the same solvent used when filling the nanoparticles), fluorescence signals are observed at both $550 \mathrm{~nm}$ and $660 \mathrm{~nm}$. Recording the fluorescence signal when the fibre is filled with only the solvent is necessary to ensure that the only possible sources of 
fluorescence are either the nanoparticles themselves, or the glass itself. Indeed when the fibre is filled with toluene the recorded signal in both the red and the green drops as the mode spreads out in to the holes, and the fraction of the fluorescence that is recaptured in to the guided modes drops as the index contrast decreases [1].

\section{CONCLUSION}

We have demonstrated that suspended-core optical fibres make a good platform both for utilizing erbium nanoparticles. The use of these nanocrystals within the fibre holes form the basis of an improved sensing architecture; by directly pumping the nanocrystals in the near-IR rather than at visible wavelength. The detection limit has been reduced here to $660 \mathrm{fM}$ is a significant improvement over the $10 \mathrm{pM}$ that had previously been demonstrated using quantum dots and a visible laser in a similar fibre. In the $29 \mathrm{~nL}$ volume of the fibre this corresponds to approximately 11000 particles within the holes. This shows that these particles have strong prospects for highly sensitive detection when used as labels for biological agents. Alternatively, these molecules could find use as a pump source for conventional organic fluorophores [11], avoiding the majority of the autofluorescence emission [1] typically present in these glasses when using visible excitation. Due to the close proximities of the particles and fluorophores the efficiency of fluorescence generation has been shown to be high using this technique [11].

As such the use of nanoparticles as fluorescent labels in suspended core fibres should enable improved detection, with a reduction in the minimum detectable concentration compared to what has previously been demonstrated in fibre.

\section{ACKNOWLEDGEMENTS}

We would like to acknowledge Zhenda Lu and A/Prof. Yadong Yin, from University of California, Riverside for the supply of nanoparticles, Roger Moore from the University of Adelaide for fibre fabrication and Mai-Chi Nguyen for assistance with the measurements. T. M. Monro acknowledges the support of an ARC Federation Fellowship. D. Jin wishes to acknowledge his ARC postdoctoral fellowship award. We acknowledge Schott Glass Co. for the supply of F2HT glass.

\section{REFERENCES}

[1] E. P. Schartner, H. Ebendorff-Heidepriem, S. C. Warren-Smith, R. T. White, and T. M. Monro, "Driving down the Detection Limit in Microstructured Fiber-Based Chemical Dip Sensors,” Sensors, 11(3), 2961-2971 (2011).

[2] C. Cordeiro, M. Franco, G. Chesini, E. Barretto, R. Lwin, C. Brito Cruz, and M. Large, "Microstructured-core optical fibre for evanescent sensing applications," Opt. Express, 14(26), 13056-13066 (2006).

[3] S. Wu, G. Han, D. J. Milliron, S. Aloni, V. Altoe, D. V. Talapin, B. E. Cohen, and P. J. Schuck, "Non-blinking and photostable upconverted luminescence from single lanthanide-doped nanocrystals," Proceedings of the National Academy of Sciences, 106(27), 10917 (2009).

[4] F. Wang, Y. Han, C. S. Lim, Y. Lu, J. Wang, J. Xu, H. Chen, C. Zhang, M. Hong, and X. Liu, "Simultaneous phase and size control of upconversion nanocrystals through lanthanide doping,” Nature, 463(7284), 1061-1065 (2010).

[5] R. Abdul Jalil, and Y. Zhang, "Biocompatibility of silica coated NaYF4 upconversion fluorescent nanocrystals,” Biomaterials, 29(30), 4122-4128 (2008).

[6] F. Meiser, C. Cortez, and F. Caruso, "Biofunctionalization of Fluorescent Rare Earth Doped Lanthanum Phosphate Colloidal Nanoparticles,” Angewandte Chemie International Edition, 43(44), 5954-5957 (2004).

[7] D. K. Chatterjee, A. J. Rufaihah, and Y. Zhang, "Upconversion fluorescence imaging of cells and small animals using lanthanide doped nanocrystals,” Biomaterials, 29(7), 937-943 (2008).

[8] T. Monro, S. Warren-Smith, E. Schartner, A. Francois, S. Heng, H. Ebendorff-Heidepriem, and S. Afshar V, "Sensing with suspended-core optical fibers," Opt. Fiber Technol., 16, 343-356 (2010).

[9] H. Ebendorff-Heidepriem, S. Warren-Smith, and T. Monro, "Suspended nanowires: fabrication, design and characterization of fibers with nanoscale cores,” Opt. Express, 17(4), 2646-2657 (2009).

[10] H. Ebendorff-Heidepriem, and T. M. Monro, "Extrusion of complex preforms for microstructured optical fibers,” Optics Express, 15(23), 15086-15092 (2007).

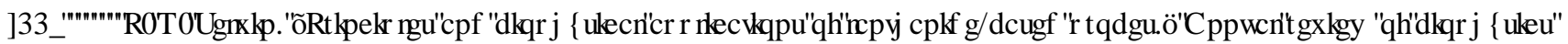

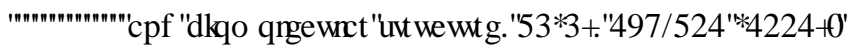

